\title{
High Strain Rate Behaviour of Auxetic Cellular Structures
}

\author{
Nejc Novak ${ }^{1, a^{*}}$, Matej Vesenjak ${ }^{1, b}$, Shigeru Tanaka ${ }^{2, c}$, Kazuyuki Hokamoto ${ }^{2, d}$, \\ Baoqiao Guo ${ }^{3, e}$, Pengwan Chen ${ }^{3, f}$ and Zoran Ren ${ }^{1,4 \mathrm{~g}}$ \\ ${ }^{1}$ Faculty of Mechanical Engineering, University of Maribor, Maribor, Slovenia \\ ${ }^{2}$ Institute of Pulsed Power Science, Kumamoto University, Kumamoto, Japan \\ ${ }^{3}$ State Key Laboratory of Explosion Science and Technology, Beijing Institute of Technology, \\ Beijing, China \\ ${ }^{4}$ IROAST, Kumamoto University, Kumamoto, Japan \\ an.novak@um.si, 'bmatej.vesenjak@um.si, 'tanaka@mech.kumamoto-u.ac.jp, \\ dhokamoto@mech.kumamoto-u.ac.jp, ${ }^{e}$ baoqiao_guo@bit.edu.cn, ${ }^{f}$ pwchen@bit.edu.cn, \\ gzoran.ren@um.si
}

Keywords: Cellular Structures, Auxetic Materials, High Strain Rate, Experimental Testing, Computational Simulations

\begin{abstract}
Auxetic cellular structures are modern metamaterials with negative Poisson's ratio. The auxetic cellular structures build from inverted tetrapods were fabricated and experimentally tested under dynamic loading conditions to evaluate the effect of strain rate on their deformation mode. The Split-Hopkinson Pressure Bar (SHPB) apparatus was used for testing at strain rates up to $1,250 \mathrm{~s}^{-1}$, while a powder gun was used for testing at strain rates up to $5,000 \mathrm{~s}^{-1}$. The homogeneous deformation mode was observed at lower strain rates, while shock deformation mode was predominant at higher rates. The results have shown that the strain rate hardening of analysed auxetic specimens is prominent at higher strain rates when the shock deformation mode is observed, i.e. when most of deformation occurs at the impact front. Relevant computational models in LS-DYNA were developed and validated. A very good correlation between the computational and experimental data was observed.
\end{abstract}

\section{Introduction}

Auxetic cellular structures are novel metamaterials with negative Poisson's ratio - they tend to expand in lateral direction when subjected to tensile loading and vice versa in the case of compression loading [1]. This behaviour can be beneficial in many applications, especially in the crashworthiness, ballistic protection and energy absorption applications [2]. The mechanical behaviour of auxetic structures is well characterised and understood for quasi-static loading conditions, but not so much for dynamic and impact loading due to insufficient experimental characterisation attempts so far. Past studies were mostly concerned with the quasi-static elastic behaviour of uniform auxetic structures at very small strains [3] and limited ballistic resistance study [4]. Mechanical behaviour of some particular auxetic structures was characterised by uniaxial quasi-static compressive and tensile tests [5-10]. The Split-Hopkinson Pressure Bar (SHPB) experiments were also carried out for auxetic cellular structures fabricated with additive manufacturing, including also polymer fillers [11]. There is a clear need to test the auxetic cellular structures also under high strain rate loading conditions to comprehensively evaluate their behaviour also at highly dynamic loading. Especially since there are many applications where these metamaterials can be used efficiently.

(c) (1) Content from this work may be used under the terms of the Creative Commons Attribution 3.0 license. Any further distribution of this work must maintain attribution to the author(s) and the title of the work, journal citation and DOI. Published under license by Materials Research Forum LLC. 


\section{Specimens fabrication}

The specimens build from inverted tetrapods were used in this research. Inverted tetrapods (Fig. 1a), are assembled in a particular way to define the geometry of the investigated specimens (Fig. 1b-c). The specimen's inverted tetrapod dimensions were (Fig. 1a): $a=3.5 \mathrm{~mm}, h=3 \mathrm{~mm}, d h=$ $0.5 \mathrm{~mm}$, while the circular cross-section diameter of the struts was in range from 0.38 to 0.53 , depending on the porosity (Table 1). Two types of specimens were analysed in this work: a) short and b) long specimens. The difference between the analysed types of specimens was in length in X2 direction (Table 1). The specimens were fabricated from the Ti-6Al-4V alloy powder by the selective electron-beam melting method (SEBM) at the Institute of Materials Science and Technology (WTM), University of Erlangen-Nürnberg, Germany [12].

a)

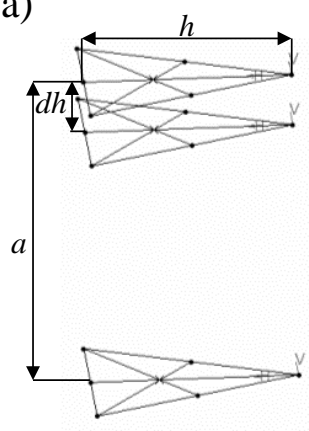

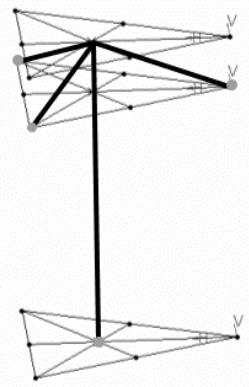

b)

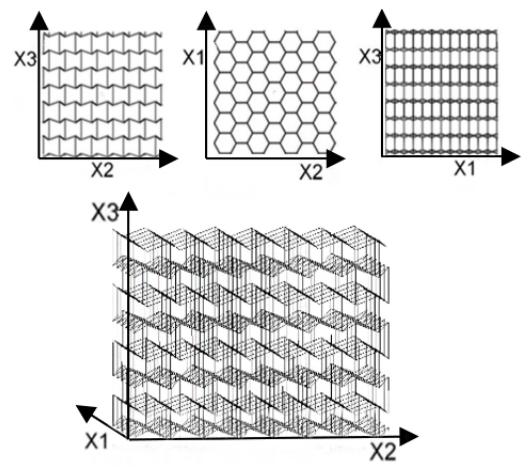

c)



Figure 1: Geometry of auxetic specimens build from inverted tetrapods: a) inverted tetrapod, b) geometry in orthogonal views and c) fabricated specimen

Table 1: Specimens data

\begin{tabular}{|c|c|c|c|c|}
\hline \multicolumn{5}{|c|}{ Short specimens } \\
\hline & $\begin{array}{l}\text { Dimensions [mm] } \\
|\mathrm{X} 1| \times|\mathrm{X} 2| \times|\mathrm{X} 3|\end{array}$ & $\begin{array}{c}\text { Mass (std. dev.) } \\
{[\mathrm{g}]}\end{array}$ & $\begin{array}{l}\text { Density } \\
{\left[\mathrm{g} / \mathrm{cm}^{3}\right]}\end{array}$ & $\begin{array}{c}\text { Porosity } p \\
{[-]}\end{array}$ \\
\hline Middle porosity & $15.6 \times 19.2 \times 18.7$ & $3.45(0.023)$ & 0.62 & 0.86 \\
\hline \multicolumn{5}{|c|}{ Long specimens } \\
\hline & $\begin{array}{l}\text { Dimensions [mm] } \\
|\mathrm{X} 1| \times|\mathrm{X} 2| \times|\mathrm{X} 3|\end{array}$ & $\begin{array}{c}\text { Mass (std. dev.) } \\
\text { [g] }\end{array}$ & $\begin{array}{l}\text { Density } \\
{\left[\mathrm{g} / \mathrm{cm}^{3}\right]}\end{array}$ & $\begin{array}{c}\text { Porosity } p \\
{[-]}\end{array}$ \\
\hline High porosity & \multirow{3}{*}{$15.6 \times 40.5 \times 18.7$} & $4.67(0.138)$ & 0.40 & 0.91 \\
\hline Middle porosity & & $7.24(0.051)$ & 0.61 & 0.86 \\
\hline Low porosity & & $9.04(0.075)$ & 0.76 & 0.83 \\
\hline
\end{tabular}

\section{High strain rate experimental testing}

High strain rate experimental testing was performed using two different experimental devices: a) Split Hopkinson Pressure Bar (SHPB) apparatus at the State Key Laboratory of Explosion Science and Technology, Beijing Institute of Technology, Beijing, China and b) the powder gun at the Institute of Pulsed Power Science, Kumamoto University, Kumamoto, Japan. The achieved loading velocities were $25 \mathrm{~m} / \mathrm{s}$ and $220 \mathrm{~m} / \mathrm{s}$ using the SHPB and the powder gun, respectively. The loading velocities correspond to the strain rates up to $1,250 \mathrm{~s}^{-1}$ and $10,000 \mathrm{~s}^{-1}$ for short specimens and $5,000 \mathrm{~s}^{-1}$ for long specimens, respectively. In the case of powder gun experiments the mechanical response was evaluated with the PVDF gauge (Piezo film stress gauge, PVF2 11125EK, Dynasen), as in previous experiments described by Tanaka et al. [13]. A homogenous 
deformation mode of short auxetic specimens was observed (similar to quasi-static loading conditions) behaviour during the SHPB loading, Fig. 2 [10].

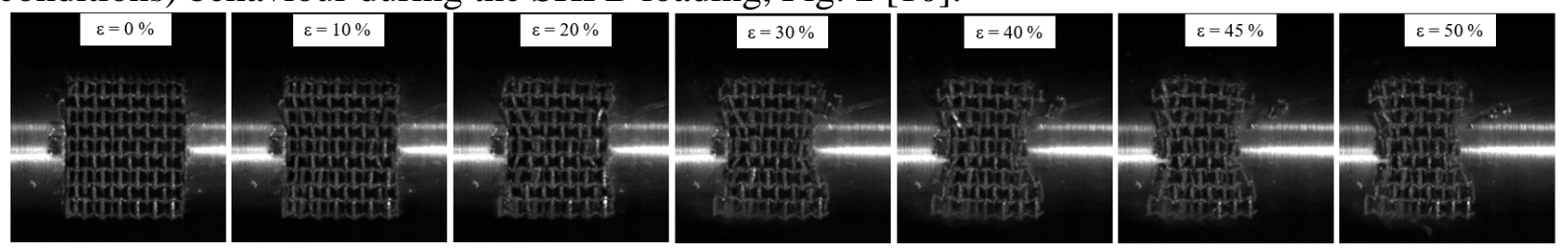

Figure 2: Deformation behaviour of short specimens in X1 direction during SHPB testing (view in the $X 2$ direction)

The typical deformation behaviour of long specimens during the powder gun experiment is shown in Fig. 3, where the crushing sequence was analysed using digital images of the highspeed camera recording. Due to high strain rates achieved $\left(10,000 \mathrm{~s}^{-1}\right.$ and $\left.5,000 \mathrm{~s}^{-1}\right)$, the shock deformation mode was observed in all tested specimens with various porosities. The main deformation of specimens throughout the tests (up to very large deformations) was concentrated only at the area, where the specimens impacts the fixed rigid plate.
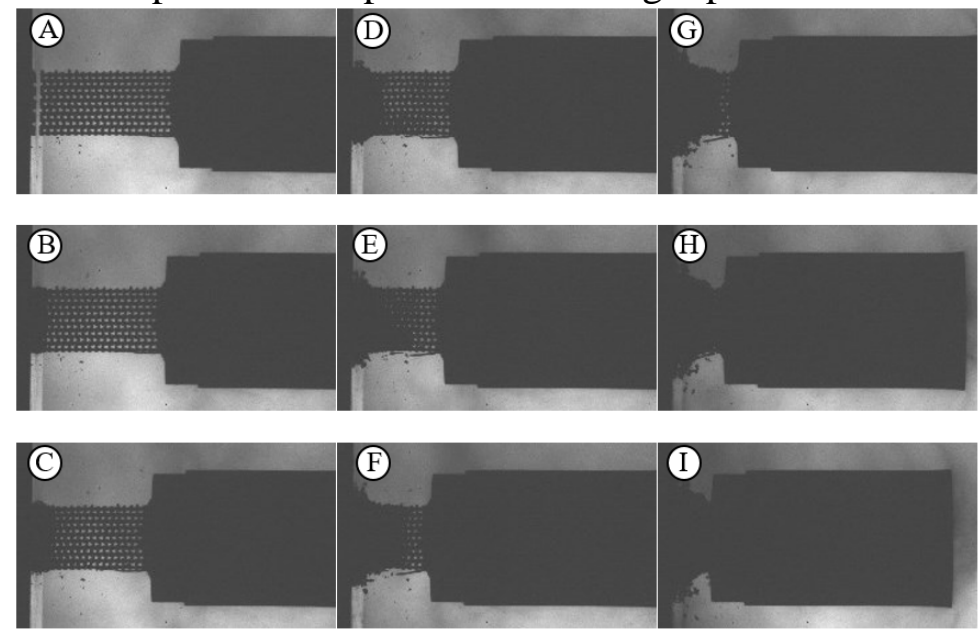

Figure 3: Deformation behaviour of long specimens with middle porosity in X2 direction during powder gun testing

The average pressure (stress) measurements recorded by the gauge at the fixed rigid measuring plate for long specimens with all three porosities and their comparison to quasi-static results are shown in Fig. 4. A signal filter with moving point average (10 data points) was used to smooth higher frequency response in recorded experimental data. As it can be observed from Fig. 4, the change of deformation mode (from homogenous mode under quasi-static loading to shock mode under high strain rate loading) causes large strain rate hardening in comparison to the results of quasi-static tests (with a strain rate of $0.005 \mathrm{~s}^{-1}$ ) for identical auxetic specimens [10]. 


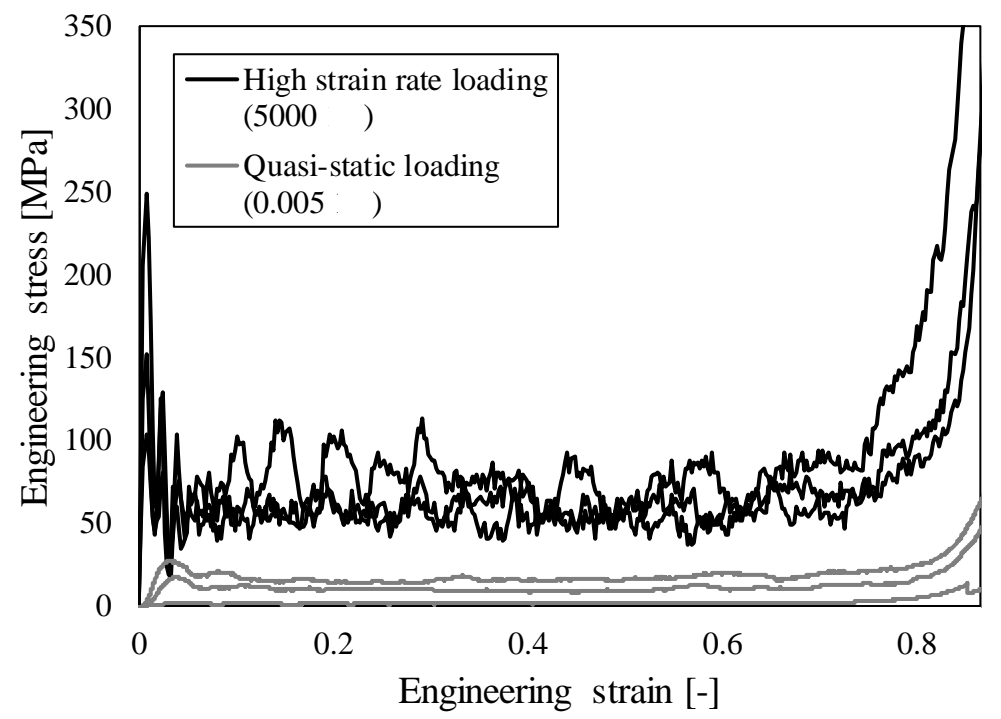

Figure 4: Comparison of quasi-static and high strain rate loading responses of long auxetic cellular structures with different porosities

\section{Computational simulations}

The relevant computational models were developed using the LS-DYNA software and validated. The elasto-plastic material model with damage and failure (MAT_024) was validated using the above reported experimental results. The relevant material parameters for the applied material model are the following: density $\rho=4430 \mathrm{~kg} / \mathrm{m}^{3}$, Young's modulus $E=120000 \mathrm{MPa}$, Poisson's ratio $v=0.3$, initial yield stress $\sigma_{\text {yield }}=1300 \mathrm{MPa}$, the definition of linear hardening with the second point in stress-strain diagram $\left(\sigma_{2}, \varepsilon_{\mathrm{pl}, 2}\right)-(1800 \mathrm{MPa}, 0.8)$ and the critical strain $\varepsilon_{\text {crit }}=0.15$ at the start of material damage. Further details about computational modelling can be found in previously reported work $[10,14]$.

The experimental results obtained by SHPB were used only for visual comparison of deformation behaviour. An excellent agreement between the experimental and computational results can be observed from Fig. 5.
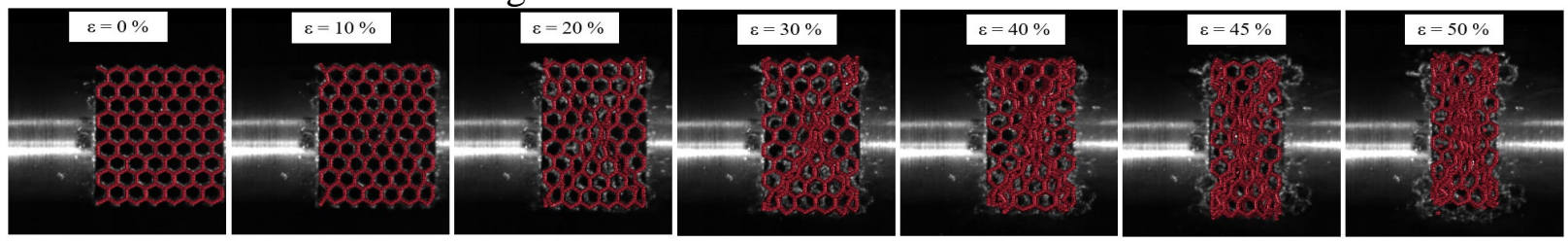

Figure 5: Comparison of experimental and computational results of SHPB loading of short specimens in $X 1$ direction (view in the direction $X 3$ )

The visual comparison of computational and experimental results for the case of powder gun loading is shown in Fig. 6, where good agreement can be observed as well. The computational model predicts the change of deformation mode from homogeneous to shock mode successfully.

The results from the computational models were further compared with experimental results in terms of engineering stress (Fig. 7), where a good correlation was noted. The mechanical properties (e.g. densification strain and plateau stress) are reproducible in the case of low and middle porosity, while in the case of high porosity, a larger deviation can be observed. This phenomenon was already explained and justified in previous work [12], where the consequence of the fabrication procedure and resulting imperfections was discussed in detail. 


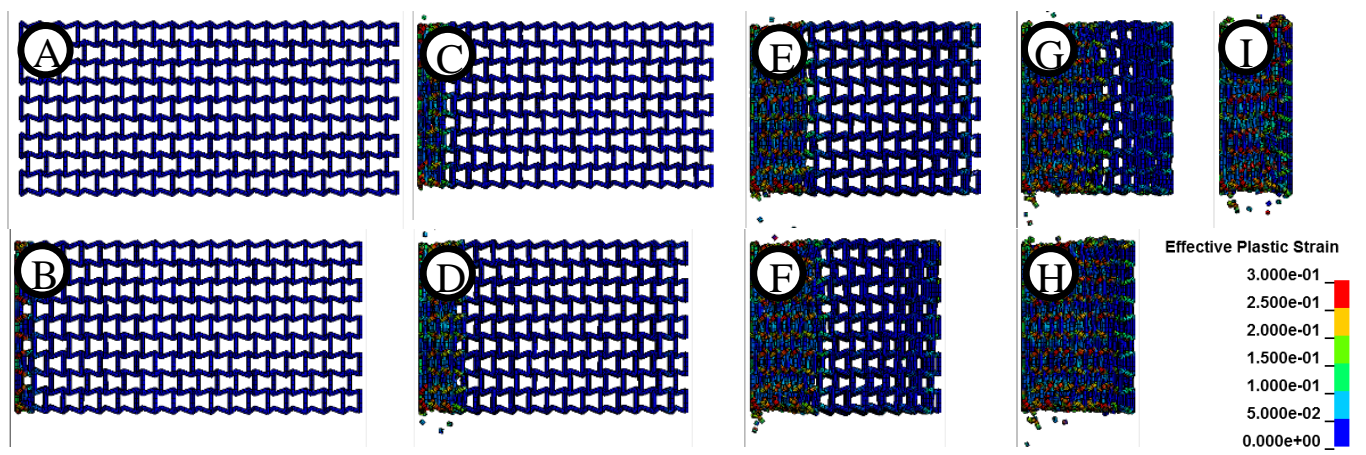

Figure 6: Deformation behaviour of long specimens with middle porosity in X2 direction in powder gun testing



Figure 7: Comparison of experimental and computational results of powder gun loading of long specimens with different porosities

\section{Summary}

The auxetic cellular structures build from inverted tetrapods were analysed in this work. The high strain rate deformation response was evaluated with experimental testing using the SHPB apparatus and the powder gun device. The homogeneous deformation mode was observed in the case of SHPB testing at strain rates up to up to $1,250 \mathrm{~s}^{-1}$. In the case of powder gun loading at strain rates up to up to10,000 $\mathrm{s}^{-1}$ the deformation mode changes to shock mode, where most of the material is deformed at the impact front. The change of deformation mode causes large strain rate hardening effect of all analysed auxetic structures, which significantly influences the mechanical response. The relevant computational models were developed and successfully validated.

\section{Acknowledgement}

The research was performed within the framework of the basic research project J2-8186 entitled "Development of multifunctional auxetic cellular structures", which is financed by the Slovenian Research Agency "ARRS". The authors acknowledge the financial support from the Slovenian Research Agency (research core funding No. P2-0063). This paper is supported by the opening project of State Key Laboratory of Explosion Science and Technology (Beijing Institute of Technology) - opening project number KFJJ17-03M. 


\section{References}

[1] K. E. Evans, M. A. Nkansah, I. J. Hutchinson, and S. C. Rogers, "Molecular Network Design,” Nature, vol. 353, p. 124, 1991. https://doi.org/10.1038/353124a0

[2] N. Novak, M. Vesenjak, and Z. Ren, “Auxetic cellular materials - a Review,” Strojniški Vestn. - J. Mech. Eng., vol. 62, no. 9, pp. 485-493, 2016. https://doi.org/10.5545/svjme.2016.3656

[3] T. Bückmann et al., "Tailored 3D Mechanical Metamaterials Made by Dip-in DirectLaser-Writing Optical Lithography,” Adv. Mater., vol. 24, no. 20, pp. 2710-2714, 2012. https://doi.org/10.1002/adma.201200584

[4] C. Qi, S. Yang, D. Wang, and L.-J. Yang, "Ballistic resistance of honeycomb sandwich panels under in-plane high-velocity impact,” Sci. World J., vol. 2013, no. SEPTEMBER 2013, p. 892781, 2013. https://doi.org/10.1155/2013/892781

[5] E. A. Friis, R. S. Lakes, and J. B. Park, "Negative Poisson's ratio polymeric and metallic foams,” J. Mater. Sci., vol. 23, no. 12, pp. 4406-4414, 1988. https://doi.org/10.1007/bf00551939

[6] S. Mohsenizadeh, R. Alipour, M. Shokri Rad, A. Farokhi Nejad, and Z. Ahmad, "Crashworthiness assessment of auxetic foam-filled tube under quasi-static axial loading," Mater. Des., vol. 88, pp. 258-268, Dec. 2015. https://doi.org/10.1016/j.matdes.2015.08.152

[7] S. Hou, T. Liu, Z. Zhang, X. Han, and Q. Li, "How does negative Poisson's ratio of foam filler affect crashworthiness?,” Mater. Des., vol. 82, pp. 247-259, Oct. 2015. https://doi.org/10.1016/j.matdes.2015.05.050

[8] L. Yang, O. Harrysson, H. West, and D. Cormier, "Compressive properties of Ti-6Al-4V auxetic mesh structures made by electron beam melting," Acta Mater., vol. 60, no. 8, pp. 3370-3379, 2012. https://doi.org/10.1016/j.actamat.2012.03.015

[9] L. Yang, O. Harrysson, H. West, and D. Cormier, "Mechanical properties of 3D re-entrant honeycomb auxetic structures realized via additive manufacturing,” Int. J. Solids Struct., vol. 69-70, pp. 475-490, Sep. 2015. https://doi.org/10.1016/j.ijsolstr.2015.05.005

[10] N. Novak, M. Vesenjak, L. Krstulović-Opara, and Z. Ren, "Mechanical characterisation of auxetic cellular structures built from inverted tetrapods,” Compos. Struct., vol. 196, pp. 96-107, 2018. https://doi.org/10.1016/j.compstruct.2018.05.024

[11] T. Fíla et al., "Impact Testing of Polymer-filled Auxetics Using Split Hopkinson Pressure Bar,” Adv. Eng. Mater., p. n/a--n/a, 2017. https://doi.org/10.1002/adem.201700076

[12] J. Schwerdtfeger, P. Heinl, R. F. Singer, and C. Körner, “Auxetic cellular structures through selective electron beam melting,” Phys. Status Solidi B, vol. 247, no. 2, pp. 269272, 2010. https://doi.org/10.1002/pssb.200945513

[13] S. Tanaka et al., "High-velocity impact experiment of aluminum foam sample using powder gun,” Meas. J. Int. Meas. Confed., vol. 44, no. 10, pp. 2185-2189, 2011. https://doi.org/10.1016/j.measurement.2011.07.018

[14] N. Novak, K. Hokamoto, M. Vesenjak, and Z. Ren, "Mechanical behaviour of auxetic cellular structures built from inverted tetrapods at high strain rates," Int. J. Impact Eng., vol. 122, pp. 83-90, 2018. https://doi.org/10.1016/j.ijimpeng.2018.08.001 